\title{
Guided and Unguided Student Reflections
}

\author{
Amanda Matheson, ${ }^{1}$ Laura Wood, ${ }^{2}$ Elizabeth Hane, ${ }^{3}$ and Scott Franklin ${ }^{4}$ \\ ${ }^{1}$ Department of Physics, Colorado School of Mines, Golden, CO 80401 \\ ${ }^{2}$ Department of Physics, Seattle Pacific University, Seattle, WA 98119 \\ ${ }^{3}$ Gosnell School of Life Sciences, RIT, Rochester, NY 14623 \\ ${ }^{4}$ School of Physics and Astronomy, RIT, Rochester, NY 14623
}

\begin{abstract}
Self-reflection is important metacognitive skill, enabling students to build coherence into their learning and embed content in a broader context. While various pedagogical techniques exist to encourage student reflection, little research has examined the differences between formally guided, partially guided and unguided reflections. This study focuses on student responses to online Guided Reflection Forms (GRFs) from students in a firstsemester non-physics class and, separately, a sophomore-level Vibrations \& Waves course for physics majors, and compares these guided reflections with partially guided and unguided journals from a STEM enrichment summer program for incoming college students. A previously developed coding scheme was used on guided reflections and the LIWC computational linguistics tool used to confirm the distinct nature of the categories. A new coding scheme was created and validated for the unguided journals. We find that both guided and unguided reflections elicit metacognitive and reflective practice, although of measurably different frequencies and kinds.
\end{abstract}

\section{INTRODUCTION}

A number of studies (e.g. [1-4]) have found metacognitive reflection and journaling to increase student performance and understanding. These need not be directly related to content; Mason [5] found that students who set specific goals about future growth, identified theory and tools to achieve these goals, and reflected regularly on progress toward those goals demonstrated greater well-being and large improvements in overall grades. Journals can encourage students to reflect on their beliefs, thoughts, and actions, in the process increasing self-efficacy and important sense of self.

Reflections in a classroom setting can be either guided by explicit questions or prompts [6] or unguided. Unstructured journals run the risk of being used as a simple log book or checklist, $[7,8]$ or subject to student beliefs that affective or personal reflections are inappropriate in an academic setting. Guided reflections may help students increase their reflective sophistication; Kori [4] found that, after working through a scaffolded reflection that built upon each step of an experiment, students moved from simpler descriptions to more sophisticated justifications and critiques of their experimental methods and analyses.

There have been few attempts to systematically characterize guided or unguided journals, and virtually no attempts to compare the two. Dounas-Frazer and Reinholz [6] developed an online Guided Reflection Form that encourages specific reflections on weekly content. In addition, they developed a rubric for codifying student statements, finding students forthcoming with simple narrative statements but struggling to articulate concrete plans to overcome setbacks. Kori [4] developed a similar categorization scheme for his work on elementary and high-school student responses to open-ended, yet prompted questions, although his work was limited to students' understanding of their reasoning process and did not include personal or non-academic experiences.

\section{STUDY DESIGN}

\section{A. Instructional Contexts}

Guided Reflection Forms (GRFs), adapted from [6] were completed weekly by forty-one first-year STEM majors taking the introductory level "Metacognitive Approaches to Scientific Inquiry" (Metacognition) and thirty-eight physics and engineering majors enrolled in the sophomore-level "Vibrations \& Waves" $(V \& W)$ course. GRFs prompt students to answer a series of three questions:

1. Describe an experience from the past week that you would like to improve upon in the future,

2. Select strategies that you used to overcome the difficult situation, and

3. Describe how you would improve upon the experience in the future.

Over the course of the semester, this yielded 399 individual submissions from the Fall 2015 section of Metacognition and 271 submissions from the Spring 2016 section of $V \& W$. Each submission received a personal e-mail response from the instructor that affirmed the student's reflections and offered suggestions and resources to help the student implement the desired improvements.

The Metacognition students were all first-generation or deaf/hard of hearing incoming STEM majors taking part in a program, Integrating Metacognitive Processes and Research to Ensure Student Success (IMPRESS), [9] designed to increase retention. The course introduces students to a variety of metacognitive and affective topics including mindset, selfassessment, stereotype threats, and the impacts of micro- and macro-aggressions. The course is co-taught by an environmental scientist and a physicist (the last author); the physicist also taught $V \& W . V \& W$ is taught in an active learning setting, and covers traditional content from [10]. 
The IMPRESS program includes a two-week summer program for deaf/hard-of-hearing (DHH) and first generation incoming STEM majors. Twenty participants conduct experiments and develop models for climate change as context for exploring and developing metacognitive and reflective practices. Journals from IMPRESS 2014 and 2015 were examined as part of this study. In 2014, journals were unguided, with students only shown examples of topics they could write about. In 2015, students were shown the following set of questions, adapted from the Guided Reflection Forms:

- What do you think you learned about thinking style during this exercise?

- What mental resources did you use during the exercise?

- Think of a specific mental skill you could improve on that would you in this exercise.

- Describe the kinds of skills you think would help you make the above improvements.

-What strategies did you notice your classmates using?

- How did you group members' strategies impact your approach to the problem?

The 2014 IMPRESS journals are therefore considered unguided, with only suggestions made of questions the students could answer, whereas 2015 journals are almost (but not quite) as guided as GRFs.

Students completed their journals each evening in a diarystyle notebook (e.g. not a lab notebook). Importantly, they did not have the prompts in front of them when responding. As a result, students took different approaches to completing their journals. Some would number each response and answer in order, while others wrote complete paragraphs that integrated their responses to all the questions. Students did not receive feedback on their journals in either year.

The different forms of filling out reflections - online forms with prompts versus blank paper journals without significantly affected the level of guidance, with the GRFs the most guided of the three methods examined.

\section{GUIDED REFLECTIONS}

GRF submissions were randomly scrambled to prevent the possibility of bias, with all name, date and course data removed. Two researchers separately coded each statement by the coding rubric developed by [6] (see Table I). Together, these categories accounted for $40 \%$ of all student statements in both Metacognition and $V \& W$.

GRF codes were entered into NVivo, and an Inter-Rater Reliability test conducted. Given the low coding density per response, a Kappa coefficient within the range of 0.4-0.7 was considered satisfactory, with $\kappa>0.7$ representing nearly perfect agreement. Any categories with $\kappa<0.6$ were discussed
TABLE I. GRF analysis codes developed in [6]

\begin{tabular}{|l|l|}
\hline Coding Category & Description \\
\hline Narrative & literal descriptions of events \\
\hline Growth & broad goals for improvement \\
\hline Action & specific goals/steps for improvement \\
\hline Achievement & specific desired achievements \\
\hline
\end{tabular}

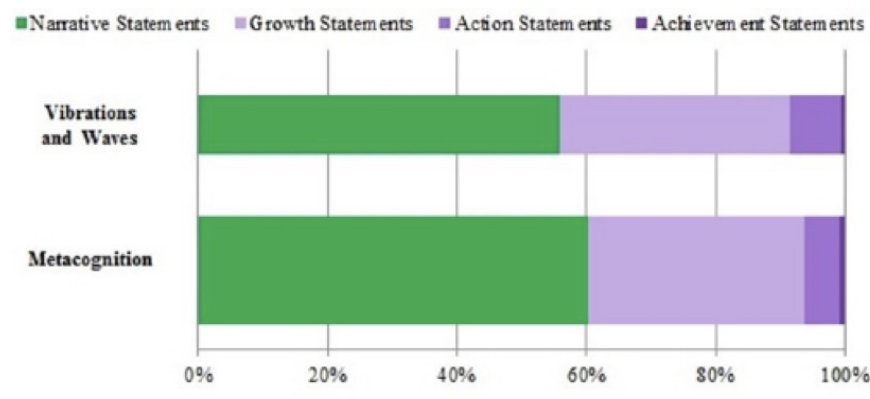

FIG. 1. Frequency of sentences of different types, normalized by total number of coded sentences. The introductory level Metaocgnition course and the sophomore-level Vibrations \& Waves physics course show nearly identical prevalence of narrative statements and scarcity of Action statements.

until an agreement was reached. When all the GRFs were considered together, $\langle\kappa\rangle=0.6$, an acceptable value for data with low coding density.[11]

\section{A. Analysis: coding}

The first comparison is between the guided reflections of incoming STEM majors in the Metacognition course with the second-year physics majors in Vibrations \& Waves. A ttest of the frequency of coded statements by week and across the entire semester revealed no statistically significant differences between the two sets of statements.

The distribution of coded statement frequency in the two groups is shown in Fig. 1. Both groups of student reflections are dominated by narrative statements, which make up almost $60 \%$ of all coded statements. This is followed by broad growth statements, which comprise almost $30 \%$ of all statements. Very few of the statements $(<20 \%)$ are either specific action or achievement statements. This supports the more general conclusion that students prefer to articulate less specific goals and narratives and struggle to generate concrete plans to address obstacles that arise.

There is evidence that GRF prompts elicit the desired types of responses. $87 \%$ of responses to the prompts "Is there anything else that you would like to share," and "Describe a specific experience from last week that you would like to improve upon" were Narrative statements. Similary, $81 \%$ of responses to the prompts "Comment on your experience using these resources last week" and "Describe an aspect of 
TABLE II. LIWC category scores for statements coded as Narrative, Growth, Action and Achievement. Shaded boxes indicate scores significantly different from the overall mean. Each code has a distinct combination of LIWC category scores, validating the coding as unique and differentiatable.

\begin{tabular}{|l|c|c|c|}
\hline Coding Category & Analytic & Authenticity & Tone \\
\hline Narrative & 53 & 96 & 24 \\
\hline Growth & 68 & 90 & 71 \\
\hline Action & 69 & 99 & 43 \\
\hline Achievement & 72 & 67 & 71 \\
\hline
\end{tabular}

this experience that you can improve in the future" were goal statements. Action and Achievement statements are seen in roughly equal proportion in response to goal-inducing questions. These results are comparable to those seen in [6].

\section{B. Analysis: Linguistic Inquiry and Word Count (LIWC)}

In recent years, computerized linguistic analysis, in which collections of text are compared with established lexicons, has become more accessible. Tausczik and Pennbaker [12] have created an online tool, the Linguistic Inquiry and Word Count (LIWC) [13] that automates the procedure. LIWC draws on dominant theories in psychology, business and medicine to correlate language with psychological state. It thus allows the researcher to characterize text by emotion, thinking style and social concerns.

We analyzed all student reflections with LIWC to see if the coding rubric could be characterized with distinct LIWC "fingerprints." Three LIWC categories - Analytical Thinking, Authenticity and Emotional Tone - showed statistically significant differences across categories. Analytical thinking reflects the degree of formality, logic and hierarchical thinking. Authenticity evaluates the degree of honesty, personal investment and disclosure. Emotional tone is correlated with style: positive, upbeat language is characterized as a high emotional tone, while anxiety, sadness or hostility receives a lower score. (A fourth category, Clout, reflecting confidence and expertise was not present in our data.) Each category is rated on a scale from $0-100$, with 50 representing neutrality.

Table II shows the LIWC coding score for the collective statements in the four categories, and suggests the following classifications. Narrative statements are marked by a slightly lower Analytic score, as they are more personal recollections, and a distinctly lower (more negative) Tone. This is explained through the GRF prompt, which asks students to reflect on events or situations that they would like to improve. While the Narrative fingerprint has an obvious interpretation, the others are less intuitive. Action statements are characterized by lower Tone, although not as low as the Narratives, which suggests that students either are unsure of how to proceed or are pessimistic at their chances of successfully taking these actions. Achievement statements, which put forth specific and desired achievements, have similar reason and tone as oth-
TABLE III. Coding rubric for unguided and semi-guided journals, in order of nominally increasing metacognitive sophistication.

\begin{tabular}{|l|l|}
\hline Coding Category & Description \\
\hline Description & $\begin{array}{l}\text { narration of an activity accompanied by little } \\
\text { to no opinions or evaluations }\end{array}$ \\
\hline Logic/rationale & $\begin{array}{l}\text { explicit or implied statements of why a strat- } \\
\text { egy or approach was chosen }\end{array}$ \\
\hline Evaluation & $\begin{array}{l}\text { opinions of an activity or choice of strategy, } \\
\text { accompanied with backing reasons }\end{array}$ \\
\hline Discussion & describing alternative solutions \\
\hline Patterns of thought & $\begin{array}{l}\text { metacognitive descriptions of mental patterns } \\
\text { or strengths or weaknesses }\end{array}$ \\
\hline
\end{tabular}

ers, but lower authenticity scores. This is quite surprising, and suggests students struggle to articulate genuine goals. Finally, Growth statements, which are broader, less specific goals, are characterized by generally positive scores on all sub-categories.

\section{UNGUIDED JOURNALS}

Shifting attention from the formal classroom, we now look at student journals from the two-week pre-matriculation summer experience. Twenty journals from each of two year's (2014 \& 2015) experiences were collected. Journal entries from the summer focused on metacognitive aspects of student experiences and reflections, with less attention paid to overcoming setbacks and long-term goals. The GRF coding rubric was significantly modified to account for the different reflections seen in the journals. As part of this process, two researchers coded journal entries using the existing GRF scheme and then discussed the suitability and made agreedupon changes.

The characterization scheme was also inspired by [4], whose scheme forms a loose hierarchy of metacognitive sophistication. Codes are shown in Table III, ordered in increasing sophistication. Unguided and semi-guided journals resulted in a much wider range of topics than the formal GRFs, and may include discussions of other people's approaches or the program activity. To account for these different statements, the new coding scheme is considerably broader than that applied to GRFs.

The journals were coded in the same manner as the GRFs - each researcher coding two thirds - with an average Kappa coefficient of 0.82 for the 2014 journals and 0.63 for the 2015 journals.

\section{A. Analysis}

For the 2014 journals, students were given no explicit instructions in what to write. In 2015, students were given prompts adapted from the GRFs. Perhaps not surprising, the 2015 journals were shorter and more focused than the 
2014 journals. While many students wrote lengthy reflections including many anecdotal stories of life outside of the IMPRESS program in 2014, the same was not true in 2015. In addition to being shorter, the 2015 journals contained a statistically significant smaller proportion coded statements.

The fraction of Narrative statements in the 2015 journals is also lower than that in the 2014 journals (47\% vs. 65\%). Descriptive statements are objective recollections of events, and are thus analogous with GRF Narrative statements. Recalling that $58 \%$ of statements in the formal GRFs were Narrative, it is surprising tha that the journal prompts produce a result significantly different from the GRFs. The 2015 journals also contains more Evaluation, Patterns of Thought and Discussion statements, and fewer Logic statements.

There is evidence that the student journals from both sets are considerably richer than the classroom GRFs. More than $80 \%$ of journals (in both sets) contained statements that were coded in four of the five five categories, and $60 \%$ had statements that encompassed all five categories. Conversely, only $50 \%$ of GRFs had statements in at least three of the four cagetories, and only $4 \%$ had statements in all four. The explicit prompts in the Guided Reflection Forms, perhaps combined with the explicit classroom framing, resulted in reflections that were more constrained and less personal.

\section{CONCLUSIONS}

In this paper, we have examined different methods at encouraging student reflections. We find that online Guided Reflection Forms are successful at eliciting reflections, with little difference seen in courses of different level or student population. Prompts succeed in helping students focus, stay on topic, and engage in metacognitive reflection.

Online GRFs present are an efficient mechanism to prompt, collect and respond to student reflections. Nevertheless, large-scale analysis and synthesis remains time-consuming. We apply a new tool to this task, Linguistic Inquiry and Word Count (LIWC) discourse analysis, and find that the previously developed and validated codes can be uniquely characterized. Different codes are captured by distinctly different affective words, varying in degree of formality, personal investment, and tone. LIWC makes it possible to now study a variety of new, interesting questions, such as changes in student reflection and emotional state (as reflected in GRFs) over the course of a semester, year or academic career.

Journals outside the formal classroom environment, particularly when unguided, produce a different type of reflection, one considerably more diverse in types of statements. Unguided journals are longer, with students exploring a wider range of topics and, surprisingly, giving more narration. In both formal and informal environments, prompts are effective at focusing student reflections to desired topics, and open the possibility for online scaffolding exercises that lead to greater metacognitive awareness and associated curricular gains.

Future work could proceed along two different directions. A key feature of GRFs is timely and personalized instructor feedback. This feedback is also a rich data set that can be analyzed for linguistic characteristics. Correlating the nature of instructor feedback with subsequent student submissions can provide guidance for instructors looking to improve student metacognition. Separately, student reflections can be compared with classroom performance to look directly for a link between metacognitive sophistication and content learning.

\section{ACKNOWLEDGMENTS}

We gratefully acknowledge many useful discussions with Eleanor Sayre and members of PEER-Rochester, part of the Professional Development for Education Researchers series. Dimitri Dounas-Frazer and Daniel Reinholz first brought GRFs to our attention, and provided support and advice as this project developed. We are grateful for the support we received from student and faculty participants in RIT's REU in Modeling who provided guidance and support. Finally, we acknowledge support from the National Science Foundation under Grant Nos. DUE \#1359262 and DUE \#1317450.
[1] R. G. Bringle and Julie A. Hatcher, Educational Horizons. 179 (1999).

[2] M. Scott, T. Stelzer and G. Gladding, Physics Education Research Conference, 188 (2007).

[3] J. Westberg and H. Jason, Fostering Reflection and Providing Feedback : Helping Others Learn from Experience. New York, US. $61 * 2001)$.

[4] K. Kori, M. Maeots and M. Pedaste, Procedia - Social and Behavioral Sciences, 112, 242 (2014).

[5] A. Mason and C. Singh, AIP Physics Education Research Conference Proceedings, 1179, 197 (2009).

[6] D. R. Dounas-Frazer and D. L. Reinholz, D. L. American Journal of Physics, 83 (10), (2015).
[7] D. Boud and D. Walker, Studies in Higher Education 23, 191 (1998).

[8] P. Prinsloo, S. Slade and F. Galpin Open Learning: The Journal of Open, Distance and e-Learning, 26, 27 (2011).

[9] S. V. Franklin, E. Hane, M. B. Kustusch, C. Ptak and E. C. Sayre, "Improving retention through metacognition: a program for deaf/hard-of-hearing and first generation STEM college students." in preparation (2017)

[10] A. P. French, Vibrations and Waves, Taylor \& Francis (1971).

[11] D. Hammer and L. K. Berland, Journal of Learning Sciences, 23, 37 (2014)

[12] Y. R. Tausczik and J. W. Pennebaker, Journal of Language and Social Psychology 29, 24 (2010)

[13] https://liwc.wpengine.com 